\title{
Scalings of the tidally induced bottom boundary layer in a shallow sea under a surface heating
}

\section{AUTHOR(S):}

Akitomo, Kazunori; Hirano, Masahiro; Kinugawa, Yuya; Sakamoto, Kei; Tanaka, Kiyoshi

\section{CITATION:}

Akitomo, Kazunori ...[et al]. Scalings of the tidally induced bottom boundary layer in a shallow sea under a surface heating. Journal of Oceanography 2016, 72: 541-552

\section{ISSUE DATE:}

2016-08

URL:

http://hdl.handle.net/2433/216244

\section{RIGHT:}

(c) The Oceanographic Society of Japan and Springer Japan 2016; The final publication is available at Springer via http://dx.doi.org/10.1007/s10872-015-0343-z;; The full-text file will be made open to the public on 01 August 2017 in accordance with publisher's 'Terms and Conditions for Self-Archiving'; この論文は出版社版でありません。引用の際に は出版社版をご確認じ利用ください。; This is not the published version. Please cite only the published version. 
JO manuscript No.

(will be inserted by the editor)

1 Scalings of the tidally-induced bottom boundary layer in a

2 shallow sea under a surface heating

3 Kazunori Akitomo • Masahiro Hirano • Yuya

4 Kinugawa - Kei Sakamoto and Kiyoshi

5 Tanaka

6 Received: date / Accepted: date

K. Akitomo

Department of Geophysics, Graduate School of Science, Kyoto University, Sakyoku, Kyoto,

Japan 606-8502first address

Tel.: +81-75-753-3921

Fax: $+81-75-753-3928$

E-mail: akitomo@kugi.kyoto-u.ac.jp

M. Hirano

Department of Geophysics, Graduate School of Science, Kyoto University, Sakyoku, Kyoto 606-8502, Japan

Y. Kinugawa

Department of Geophysics, Graduate School of Science, Kyoto University, Sakyoku, Kyoto, Japan 606-8502

K. Sakamoto

Meteorological Research Institute, 1-1 Nagamine, Tsukuba, Ibaraki 305-0052, Japan

K. Tanaka

Atmosphere and Ocean Research Institute, the University of Tokyo 5-1-5 Kashiwanoha,

Kashiwa, Chiba 277-8564, Japan 
7 Abstract We have investigated properties of the tidally-induced bottom boundary 8 layer (TBBL) in a shallow sea under a surface heating, by scale argument and 9 DNS (Direct Numerical Simulation) experiment. Applying the existing scalings of the boundary layer, it is found that the height of TBBL $H_{t b b l}$ and the efficiency

11 of tidal mixing $\epsilon$ are scaled to $\left(u_{*}^{4} H /|\sigma+f| B_{s}\right)^{1 / 3}$ and $H_{h o m} / H_{t b b l}$, respectively, 12 where $u_{*}$ is the friction velocity, $\sigma$ the tidal frequency, $f$ the inertial frequency 13 (the Coriolis parameter), $B_{s}$ the surface buoyancy flux, $H$ the water depth, and ${ }_{14} H_{h o m}=u_{*} /|\sigma+f|$ the height of TBBL in a homogeneous ocean. Results of DNS 15 experiment agree with these scalings for fairly wide ranges of $u_{*}$ (or tidal amplitude $\left.{ }_{16} U_{\text {tide }}\right), H, B_{s}$, and $|\sigma / f|$. In exceptional cases with slower Earth's rotations, weaker 17 tidal flows, and shallower water depths, turbulence occurs intermittently and the 18 scaling underestimates $H_{t b b l}$ and $\epsilon$. The efficiency of tidal mixing $\epsilon$ varies from less 19 than $1 \%$ to $7 \%$ for the experimental range. This variation can partly explain the 20 reason why the critical value of Simpson-Hunter parameter which is an index of ${ }_{21}$ the position of tidal mixing front is different from place to place around the world.

Keywords Tidally-induced bottom boundary layer $\cdot$ Turbulence $\cdot$ Tidal mixing

23 front $\cdot$ Scaling argument $\cdot$ DNS

\section{$24 \quad 1$ Introduction}

25 Tides and tidal current are predominant phenomena in shallow coastal seas to control physical and biochemical environments (e.g. Simpson and Sharples, 2012).

${ }_{27}$ Their roles includes not only the horizontal transport such as water exchange

${ }_{28}$ between coastal open seas through a narrow channel (e.g. Awaji et al., 1980), but

29 also the vertical mixing which essentially controls the primary production. 
While a summer heating stratifies the water column against tidal (wind) mixing

31 in some regions, stronger tidal mixing keeps its vertical homogeneity against the

32 heating in other regions. The so-called tidal mixing front formed in between is considered to play a crucial role in enhancing the primary production. Since it was first reported in the Irish Sea by Simpson and Hunter (1974), similar features have been detected in coastal regions throughout the world (e.g. Garrett et al., 36 1978; Pingree et al., 1978; Lie, 1989; Yanagi and Tamaru, 1990; Glorioso and 37 Flather, 1995; Kobayashi et al., 2006).

Simpson and Hunter (1974) proposed an index of the location where the tidal 39 front is formed, based on the energetic balance that the potential energy loss due to 40 a surface heating is just canceled by the turbulent kinetic energy due to tidal flow ${ }_{41}$ at the front. That is, the front is formed along isolines of the following parameter,

$$
\frac{H}{U^{3}}=\frac{8 C_{p} k \epsilon \rho}{3 \pi \alpha g Q}
$$

42 where $H$ is the water depth and $U$ the amplitude of tidal current (depth-mean tidal ${ }_{43}$ velocity), $Q$ the surface heat flux, $C_{p}$ the specific heat of seawater, $\alpha$ the thermal 44 expansion rate, $g$ the acceleration due to gravity, $k$ a constant in the quadratic 45 friction law, and $\epsilon$ the efficiency of the energy conversion from the turbulent kinetic 46 energy to the potential one (efficiency of tidal mixing).

47 Provided that parameters on the right-hand side of Equation (1) are constant 48 at least regionally, the logarithm of $H / U^{3}$ which is called Simpson-Hunter (strati49 fication) parameter ( $\mathrm{SH}$ parameter) is a useful index for the position of tidal front.

50 However, its value at the front varies by location around the world over the range 51 of $1.0 \sim 2.5$ (e.g. Simpson and Hunter, 1974; Garrett et al., 1978; Pingree et al., 52 1978; Lie, 1989; Yanagi and Tamaru, 1990; Glorioso and Flather, 1995; Kobayashi 
et al., 2006). This implies that SH parameter may not be a universal index for the location of tidal mixing front.

To consider this problem, turbulent properties of the tidally-induced bottom ${ }_{56}$ boundary layer (TBBL) is a key factor because whether a tidal front is formed ${ }_{57}$ or not depends on whether the TBBL reaches the sea surface or not. Executing 58 a DNS (Direct Numerical Simulation) experiment, Sakamoto and Akitomo (2006, $592008,2009)$ found that the efficiency of tidal mixing $\epsilon$ is not constant but varies ${ }_{60}$ depending on the tidal amplitude, frequency, and Earth's rotation as well as the ${ }_{61}$ height of TBBL itself although their experiment was done in a deep sea with an 62 initially-stratified condition. On the observational side, recent advances in obser63 vation technologies have allowed us to measure fine structures of turbulent field in 64 coastal seas (e.g. Tsutsumi and Matsuno, 2012). Nevertheless, we still know little ${ }_{65}$ about properties of TBBL because fine-scale observations as well as model studies 66 of turbulent tidal flow are not enough.

In this study, therefore, we investigate how properties of TBBL such as its 68 height and the efficiency of mixing are determined in a shallow sea under a surface${ }_{69}$ heating condition with scale argument and DNS model experiment. We first seek 70 for appropriate scalings of the TBBL applying the existing scaling argument in 71 Section 2. After that, DNS experiment is carried out to validate them in Section 723 , and we summarize and discuss findings of the present study in Section 4 . 


\section{Scalings of the TBBL}

2.1 Height of the TBBL

75 As for the height of the turbulent boundary layer in oceans as well as the atmo-

76 sphere, scaling argument has been often used under neutral and stable conditions

77 (e.g. Zilitinkevich et al., 2007; Yoshikawa, 2015). According to Zilitinkevich et

78 al. (2007), for example, the height of the planetary boundary layer $h_{E}$ in the

79 atmosphere is determined through the linear interpolation among the squared re-

so ciprocals of three fundamental scales, $h_{R}, h_{C N}$, and $h_{N S}$,

$$
\frac{1}{h_{E}^{2}}=\frac{1}{h_{R}^{2}}+\frac{1}{h_{C N}^{2}}+\frac{1}{h_{N S}^{2}} .
$$

${ }_{81} h_{R}$ is the height of the turbulent boundary layer under a neutral condition, $h_{C N}$

82 that under a stable one with the background stratification $N^{2}$, and $h_{N S}$ that under

83 a stable one with the buoyancy flux $B_{s}\left(=-\alpha Q g / \rho C_{p}\right)$, defined as follows.

$$
h_{R}=C_{R} \frac{u_{*}}{|f|}, \quad h_{C N}=C_{C N} \frac{u_{*}}{|f N|^{1 / 2}}, \quad \text { and } \quad h_{N S}=C_{N S} \frac{u_{*}^{2}}{\left|f B_{s}\right|^{1 / 2}},
$$

${ }_{84}$ where $u_{*}$ is the friction velocity, $f$ the Coriolis parameter, and $C_{R}, C_{C N}$ and

${ }_{85} C_{N S}$ are empirical constants. In the actual situation, $h_{E}$ is determined by the

${ }_{86}$ smallest one (or smaller ones) of these height depending on background conditions.

87 Analyzing the global dataset, Yoshikawa (2015) found that the surface mixed layer thickness in the ocean is scaled by the first and third terms during a spring heating

89 season.

90 Similar scaling argument must be valid for the TBBL. Executing numerical 91 experiments with a DNS model, Sakamoto and Akitomo $(2008,2009)$ comprehen92 sively investigated turbulent properties of the TBBL including its height. Accord93 ing to them, the tidally-averaged height of the TBBL under a neutral condition 
${ }_{94} H_{h o m}$ is given by

$$
H_{h o m}=C_{H} \frac{u_{*}}{|\sigma+f|},
$$

95

96

where $u_{*}$ is the tidally-averaged friction velocity, $\sigma$ the tidal frequency and $C_{H}$ the empirical constant $(\sim 0.7)$. They advanced their research to the TBBL in an initially-stratified deep ocean to suggest that the efficiency of tidal mixing $\epsilon$ may change depending on the ratio of $H_{h o m}$ to the height of TBBL.

The difference of the TBBL under a heating from other boundary layers, such as wind-driven surface mixed layers in oceans and planetary boundary layers in the atmosphere, is that the buoyancy forcing acts on the top boundary of the water column while the energy source of turbulence is located near the bottom boundary on the opposite side. In this situation, all the imposed buoyancy energy is not necessarily offset by the turbulent mixing. That is, when the TBBL does not reach the sea surface, only a part of the imposed buoyancy energy balances the turbulent mixing in the TBBL and the remaining is used to stratify the layer above the TBBL. This is a great contrast to other boundary layers where sources of buoyancy and turbulence are usually located on the same side of the fluid layer and directly compensate each other in an equilibrium state.

To seek for scalings appropriate to the TBBL in such a situation, we consider a simple model as follows (Fig. 1). Tidal current with an amplitude of $U_{\text {tide }}$, or its friction velocity $u_{*}$, flows over the insulated flat bottom under a constant surface heating (buoyancy flux $B_{s}$ ). After turbulent stirring is in equilibrium with the buoyancy input to the TBBL on the tidal average, we can consider that the vertical profile of buoyancy is unchanged whereas the total buoyancy increases with time. In this equilibrium, the tidally-averaged height of the TBBL $H_{t b b l}$ is 
also kept constant and the vertical buoyancy flux linearly decreases from $B_{s}$ at the sea surface to zero at the bottom. Then, we can define the effective buoyancy flux $B_{s}^{*}$ at the top of the TBBL by

$$
B_{s}^{*}=B_{s} H_{t b b l} / H,
$$

where $H$ is a constant water depth. Substituting this expression into Equation (3)

with $N=0$ and replacing $h_{R}$ with $H_{h o m}$, the following relation is obtained,

$$
H_{t b b l}=u_{*} / \sqrt{C_{H}^{-2}(\sigma+f)^{2}+C_{N S}^{-2}|\sigma+f| B_{s} H_{t b b l} / H u_{*}^{2}} .
$$

Solving this equation in terms of $H_{t b b l}$, we can get the scaling of the TBBL height.

Instead of doing so, we simplify this equation by estimating magnitudes of the two terms in the square root of the denominator on the right-hand side. Considering a shallow sea at mid latitudes, the factor $|\sigma+f|$ is the order of $10^{-4} \mathrm{~s}^{-1}$ for semidiurnal tides, and the factor $B_{s} H_{t b b l} / H u_{*}^{2}$ is the order of $10^{-2} \mathrm{~s}^{-1}$ with $B_{s} \sim 10^{-8} \mathrm{~m}^{2} \mathrm{~s}^{-3}\left(Q \sim 20 \mathrm{Wm}^{-2}\right), u_{*} \sim 10^{-3} \mathrm{~ms}^{-1}$, and $H_{t b b l} / H \sim 1$. Using these scales with the empirical constants $C_{H}$ and $C_{N S}$ of nearly unity (e.g. Sakamoto and Akitomo, 2008; Zilitinkevich et al., 2007), the second term in the square root must be two orders of magnitude larger than the first one. Thus, neglecting the first term and solving Equation (6) in terms of $H_{t b b l}$, we obtain as the scaling of $H_{t b b l}$,

$$
H_{t b b l}=C_{t b b l}\left(\frac{u_{*}^{4} H}{|\sigma+f| B_{s}}\right)^{1 / 3},
$$

where $C_{t b b l}=C_{N S}^{2 / 3}$.

It should be noted that this scaling includes the water depth $H$ in the numerator of the cubic root. This is because the effective buoyancy flux $B_{s}^{*}$ decreases with $H$ (see Equation (5)), and such dependency never appears in the scaling for other 
boundary layers. The buoyancy flux imposed at the sea surface becomes difficult to reach a deeper layer as $H$ increases, and then turbulent mixing can reach the level farther from the bottom. It is also worth noting that Equation (6) includes the scaling for the homogeneous ocean $\left(H_{h o m}\right)$, when $B_{s}=0$, or, in the limit of $H \rightarrow \infty$.

Another scaling is possible using the buoyancy frequency $N$ in the stratified layer formed above the TBBL. That is,

$$
H_{t b b l}=u_{*} / \sqrt{C_{H}^{-2}(\sigma+f)^{2}+C_{C N}^{-2}|(\sigma+f) N|} .
$$

This expression is similarly approximated to,

$$
H_{t b b l} \approx \frac{C_{C N} u_{*}}{|(\sigma+f) N|^{1 / 2}}
$$

Although $N$ is not an external parameter but determined as the result of interaction of buoyancy flux and tidal stirring, this scaling has the advantage that $N$ can be easily estimated from hydrographic observations. We will evaluate this scaling as well as Equation (7) by DNS experiment in Section 3.

\subsection{Efficiency of the energy conversion}

Based on the scaling of the TBBL height (Equation (7)) with scalings of the production rate of turbulent kinetic energy $\left(\mathrm{P}^{i}\right.$-term) and the conversion rate from the turbulent kinetic energy to the potential energy $\left(\mathrm{B}^{i}\right.$-term), we can obtain a scaling of the efficiency of tidal mixing $\epsilon\left(\equiv \mathrm{B}^{i}\right.$-term $/ \mathrm{P}^{i}$-term $)$ in the TBBL. Note that $\mathrm{P}^{i}$ - and $\mathrm{B}^{i}$-terms here are vertically-integrated positive amounts (tagged by superscript $(i)$ ), which are different from those in Section 3. Using basic scales 
such as $H, u_{*}, B_{s}$, and $H_{t b b l}$ with constants $C_{P}$ and $C_{B}$, the $\mathrm{P}^{i}$ - and $\mathrm{B}^{i}$-terms are formally scaled by,

$$
\mathrm{P}^{i}-\text { term }=C_{P} u_{*}^{3} \quad \text { and } \quad \mathrm{B}^{i}-\text { term }=C_{B} B_{s}^{*} H_{t b b l}=C_{B} \frac{B_{s} H_{t b b l}^{2}}{H},
$$

and the efficiency $\epsilon$ is given by the ratio of these terms as,

$$
\epsilon=C_{\epsilon}^{B P} \frac{B_{s} H_{t b b l}^{2}}{u_{*}^{3} H}
$$

where $C_{\epsilon}^{B P}=C_{B} / C_{P}$. With the aid of Equation (7), the $\mathrm{B}^{i}$-term and $\epsilon$ are rewritten with external parameters as,

$$
\mathrm{B}^{i}-\operatorname{term}=C_{B}^{\prime}\left(\frac{B_{s} u_{*}^{8}}{H|\sigma+f|^{2}}\right)^{1 / 3} \quad \text { and } \quad \epsilon=C_{\epsilon}^{\prime}\left(\frac{B_{s}}{u_{*} H|\sigma+f|^{2}}\right)^{1 / 3}
$$

where $C_{B}^{\prime}=C_{B} C_{t b b l}^{2}$ and $C_{\epsilon}^{\prime}=C_{B} C_{t b b l}^{2} / C_{P}$. This equation shows that the efficiency $\epsilon$ is not constant but increases with $B_{s}$ and decreases with $u_{*}, H$, and $|\sigma+f|$

Another expression of $\epsilon$ is possible with $H_{h o m}$ and $H_{t b b l}$ as

$$
\epsilon=C_{\epsilon} \frac{H_{h o m}}{H_{t b b l}},
$$

where $C_{\epsilon}=C_{t b b l}^{3} C_{B} / C_{H} C_{P}$. This scaling indicates a very simple relation that the efficiency $\epsilon$ is proportional to the ratio of the TBBL heights between a homogeneous and surface-heated oceans. It may be a merit in evaluating $\epsilon$ from hydrographic observations. Sakamoto and Akitomo (2009) suggested the similar dependency on the ratio $H_{h o m} / H_{t b b l}$ by DNS experiment although their experiment was executed in a deep sea with an initially-stratified condition.

When the TBBL reaches the sea surface (i.e., $\left.H_{t b b l}=H\right)$, Equation (13) says that the efficiency $\epsilon$ is proportional to $H_{h o m} / H$. It is this expression that must 
appear on the right-hand side of Equation (1) which defines SH parameter. Accordingly, the critical value of SH parameter may change depending on the variable efficiency $\epsilon$. It is interesting that the efficiency increases with $u_{*}$ when the TBBL reaches the sea surface whereas it decreases with $u_{*}$ when it does not (Equation (12)). In next section, we will examine validity of the scalings obtained here, performing DNS experiment under a constant heating for wide ranges of parameters.

\section{Validation with DNS experiment}

\subsection{Model configuration}

To validate the scalings obtained above, we execute three-dimensional experiment with DNS (direct numerical simulation) model which is the same as in Sakamoto and Akitomo (2008, 2009). The model basin is rectangular as shown in Figure 2, and the coordinate system $(x, y, z)$ is set for the $z$-axis upward. The governing equations are the momentum equation in the rotating frame, the continuity equation, and the advective-diffusive equation of buoyancy for a Boussinesq fluid under the rigid-lid approximation. That is,

$$
\begin{array}{r}
\frac{\partial \boldsymbol{u}}{\partial t}+\boldsymbol{u} \cdot \nabla \boldsymbol{u}+f \boldsymbol{k} \times \boldsymbol{u}=-\frac{1}{\rho_{0}} \nabla p+b \boldsymbol{k}+\nu \Delta \boldsymbol{u} \\
\nabla \cdot \boldsymbol{u}=0 \\
\frac{\partial b}{\partial t}+\boldsymbol{u} \cdot \nabla b=\kappa \Delta b
\end{array}
$$

where $\boldsymbol{u}=(u, v, w)$ is the velocity vector, $p$ the pressure, $f$ the Coriolis parameter, $\nu$ viscosity $\left(10^{-4} \mathrm{~m}^{2} \mathrm{~s}^{-1}\right)$, and $\kappa$ diffusivity $\left(10^{-4} \mathrm{~m}^{2} \mathrm{~s}^{-1}\right) . \boldsymbol{k}$ is the unit vector directing upward, and $\nabla$ and $\Delta$ are the three-dimensional gradient and Laplacian operators, respectively. Buoyancy $b$ is defined by $-\rho g / \rho_{0}$ where $\rho_{0}$ is reference wa- 
ter density $\left(1.027 \times 10^{3} \mathrm{kgm}^{-3}\right), \rho$ the deviation from it, and $g$ the acceleration due to gravity $\left(9.8 \mathrm{~ms}^{-2}\right)$. Although it has been reported that the horizontal component of Earth's rotation causes temporal change of turbulent properties of the TBBL within a tidal cycle (e.g. Wakata, 2013), we do not take it into account here because we focus on the tidally-averaged statistics of turbulence which are said to be not affected by the horizontal component of Earth's rotation (Sakamoto and

Akitomo, 2008).

The model domain is periodically connected in the horizontal direction $(x=0$, $L_{x}$ and $\left.y=0, L_{y}\right)$, and no-slip and free-slip conditions are imposed at the bottom $(z=0)$ and the rigid sea surface $(z=H)$, respectively. That is,

$$
\left\{\begin{array}{l}
u=v=w=0 \quad \text { at } \quad z=0, \\
\nu \frac{\partial u}{\partial z}=\nu \frac{\partial v}{\partial z}=w=0 \quad \text { at } \quad z=H .
\end{array}\right.
$$

For buoyancy, no-flux condition is imposed at the bottom, and constant flux $B_{s}$ at the sea surface, given by,

$$
\left\{\begin{array}{l}
\kappa \frac{\partial b}{\partial z}=0 \quad \text { at } \quad z=0 \\
\kappa \frac{\partial b}{\partial z}=B_{s} \quad \text { at } \quad z=H .
\end{array}\right.
$$

As is the same in Sakamoto and Akitomo (2008, 2009), the background tidal current $\boldsymbol{u}_{\text {tide }}=\left(u_{\text {tide }}, v_{\text {tide }}, 0\right)$ is imposed as the model forcing instead of sea surface elevation. Assuming the temporally oscillating pressure gradient in the $x$-direction, we analytically determine $\boldsymbol{u}_{\text {tide }}$ in order that it should turn to the major axis at every half tidal cycle (see Appendix and Sakamoto and Akitomo (2006, 2008) for the detailed derivation). $\boldsymbol{u}_{t i d e}$ is characterized by the amplitude $U_{\text {tide }}$, the frequency $\sigma$, and the vertical scale of the viscous bottom boundary layer 
$H_{\text {tide }}$ which is given by

$$
H_{\text {tide }}=\sqrt{\frac{2 \nu}{|\sigma+f|}},
$$

for the anti-clockwise tidal ellipse in the present experiment (positive $\sigma$ and negative $f$ ).

The horizontal lengths of the model domain, $L_{x}$ and $L_{y}$, are 128 in terms of $H_{\text {tide }}$ while the depth $H$ is 10 and 20 . The horizontal grid sizes, $\Delta x$ and $\Delta y$, are 1.0 in terms of $H_{\text {tide }}$ (128 grids) and the vertical one, $\Delta z$, changes from 0.02 near the bottom to 0.17 at the surface (128 grids). Time integration has been continued till the tidally-averaged statistics are unchanged under a constant heating (typically 30 tidal cycles) after several-cycle integration without heating. The last three cycles are used for analysis.

With constant $\sigma$ of $1.45 \times 10^{-4} \mathrm{~s}^{-1}$ (the period of $12 \mathrm{~h}$ ), 42 cases are carried out changing 4 parameters, $U_{\text {tide }}\left(0.0850 \sim 0.256 \mathrm{~ms}^{-1}\right), H(11.7 \sim 33.2 \mathrm{~m}), B_{s}$ (0.117 2.33 $\times 10^{-8} \mathrm{~m}^{2} \mathrm{~s}^{-3}$; equivalently, $\left.5 \sim 50 \mathrm{Wm}^{-2}\right)$, and $f\left(-0.364 \sim-2.91 \times 10^{-4}\right.$ $\mathrm{s}^{-1}$ ), shown in Table 1 . Note that $U_{\text {tide }}$ always represents the major-axis length of tidal ellipse.

The Reynolds number Re and the temporal Rossby number Rot which are defined by

$$
\operatorname{Re}=\frac{U_{t i d e} H_{t i d e}}{\nu} \text { and } \quad \operatorname{Rot}=\left|\frac{\sigma}{f}\right|
$$

range from 1410 to 4000, and from 0.5 to 4.0, respectively. Due to limited computational resources, the range of Re is rather small compared to the real oceans $\left(\operatorname{Re}=10^{5} \sim 10^{6}\right)$. Nevertheless, we believe that fundamental properties of the turbulent TBBL can be reproduced because basic properties of turbulent boundary layers such as mean currents and stresses become approximately independent 
of Re when its value exceeds $10^{3}$ (Coleman, 1999). The ellipticity of tidal current, which is defined here by the ratio between the major and minor axes, changes with Rot. It is 2 when Rot $=2$ and 0.5 , and 4 when $R_{t}=4$.

\subsection{Results}

After time integration of 30 tidal cycles under a constant heating, the turbulent kinetic energy and potential energy (buoyancy anomaly) fields get into a steadilyoscillating state (not shown). Figure 3 shows the vertical $(x-z)$ section of buoyancy anomaly $b_{V}(x, y, z, t)$ after 30 cycles in case 12 , which is defined by

$$
b_{V}=b-\frac{1}{V} \int_{V} b d V
$$

where $V$ is volume of the model domain $\left(=L_{x} L_{y} H\right)$. Note that $b_{V}$ represents only the deviation from the volumetric mean which linearly increases with time. Hereafter we use $b_{V}$ as buoyancy. Till this time, turbulent motion vigorously stirs the lower half to form the TBBL while strong stratification is established in the upper half. Undulations of isopycnals with a dominant horizontal scale of 10 20 m indicate that internal waves are excited by turbulence in the TBBL to propagate upwards.

To compare with the scalings obtained in Section 2, the statistic properties of the model TBBL are defined as follows. For a variable $X(x, y, z, t)$, its temporal mean $\bar{X}^{t}(x, y, z)$ is defined by the last 3-cycle average of $X$,

$$
\bar{X}^{t}=\frac{1}{3 T_{\text {tide }}} \int_{t_{0}}^{t_{0}+3 T_{\text {tide }}} X d t
$$

251 where $T_{\text {tide }}$ is the tidal period $(2 \pi / \sigma)$ and $t_{0}$ an arbitrary time after the steady oscillation is established (typically 27 cycles). The horizontal mean $\bar{X}^{x, y}(z, t)$ and 
253 the deviation from it $X^{\prime}(x, y, z, t)$ are defined by,

$$
\bar{X}^{x, y}=\frac{1}{L_{x} L_{y}} \int_{0}^{L_{y}} \int_{0}^{L_{x}} X d x d y \quad \text { and } \quad X^{\prime}=X-\bar{X}^{x, y}
$$

${ }_{254}$ Further, the horizontal and temporal mean $\bar{X}^{x, y, t}(z)$ is calculated by,

$$
\bar{X}^{x, y, t}=\frac{1}{3 T_{\text {tide }} L_{x} L_{y}} \int_{t_{0}}^{t_{0}+3 T_{\text {tide }}} \int_{0}^{L_{y}} \int_{0}^{L_{x}} X d x d y d t .
$$

Figures $4 \mathrm{a}$ and $4 \mathrm{~b}$ show the vertical profiles of the production rate of the turbulent kinetic energy, P-term, and the conversion rate of the turbulent kinetic energy to the potential one, B-term (solid line), in case 12, respectively, which are defined by,

$$
\begin{aligned}
& \mathrm{P}-\text { term }=\rho_{0}{\overline{\left(\frac{\partial u_{a v e}}{\partial z}{\overline{u^{\prime} w^{\prime}}}^{x, y}+\frac{\partial v_{a v e}}{\partial z}{\overline{v^{\prime} w^{\prime}}}^{x, y}\right)}}^{t}, \\
& \mathrm{~B}-\text { term }=-\rho_{0}{\overline{b_{V}^{\prime} w^{\prime}}}^{x, y, t}
\end{aligned}
$$

where $\left(u_{\text {ave }}, v_{\text {ave }}\right)=\left(u_{\text {tide }}+\bar{u}^{x, y}, v_{\text {tide }}+\bar{v}^{x, y}\right)$. The P-term is dominant in the thin layer near the bottom the thickness of which is characterized by the laminar (viscous) bottom boundary layer thickness $H_{\text {tide }}(\sim 1.7 \mathrm{~m}$ in this case). This reflects that the turbulence is mainly produced in the viscous bottom boundary layer associated with the strong vertical shear of the background tidal current. On the other hand, the B-term increases upward from the bottom to have the maximum at $z \sim 9 \mathrm{~m}$, and decreases toward the top of the domain after that. The weak local peak appearing near the top $(z \sim 15 \mathrm{~m})$ is probably due to breaking of internal waves excited by turbulence in the TBBL.

Corresponding to these profiles of the P- and B-terms, buoyancy anomaly ${\overline{b_{V}}}^{x, y, t}(z)$ is nearly constant below the height where the B-term has the maximum and increases upward till the top of the domain (Fig. 4c). Therefore, it is reasonable 
to define the height of TBBL $H_{t b b l}$ by that where the B-term has the maximum (indicated by cross in Figure 4), as in Sakamoto and Akitomo (2009). Figure 4b also shows the total buoyancy flux multiplied by $\rho_{0}, B_{f}$ (dashed line),

$$
B_{f}=-\rho_{0}{\overline{b_{V}^{\prime} w^{\prime}}}^{x, y, t}+\rho_{0} \kappa{\frac{\partial b_{V}}{\partial z}}^{x, y, t} .
$$

It decreases almost linearly with depth, validating our assumption for the present scaling (Equation (5)).

Figure 5a shows $H_{t b b l}$ for all cases against the scaling given by Equation (7) with $C_{t b b l}=0.383$, where the friction velocity $u_{*}$ is estimated from the last 3 -cycle average of the bottom stress (Table 1 ). (The friction velocity $u_{*}$ is not exactly proportional to $U_{t i d e}$, but it decreases with $B_{s}$ by up to $25 \%$ (Table 1; see subsection 3.3), showing that some attention is needed to use $U_{\text {tide }}$ as a velocity scale.) As seen in this figure, a good agreement between the experiment and scaling (correlation coefficient of 0.98) proves that the scaling by Equation (7) is valid for the height of the TBBL although it slightly underestimates $H_{t b b l}$ in some cases with Rot $_{\mathrm{t}}=4$ and $H=10 H_{\text {tide }}=11.7 \mathrm{~m}$ (solid red symbols in Fig. 5a).

The scaling based on the buoyancy frequency (Equation (9)) also exhibits a fairly good agreement (correlation coefficient of 0.92; Fig. 5b), where the buoyancy frequency $N^{2}$ is estimated from the profile of ${\overline{b_{V}}}^{x, y, t}(z)$ as,

$$
N^{2}=\frac{{\overline{b_{V}}}^{x, y, t}(H)-{\overline{b_{V}}}^{x, y, t}\left(H_{t b b l}\right)}{H-H_{t b b l}} .
$$

Whereas the agreement means validity of Equation (9) on the whole, close examination shows that the model results lie on the two different lines depending on whether the water depth $H$ is $10 H_{\text {tide }}$ (solid symbols) or $20 H_{\text {tide }}$ (open symbols) , implying the systematic change of the buoyancy frequency with $H$. Indeed, because the buoyancy flux balance of $\kappa N^{2}=B_{s} z / H$ is expected to be established in 
the stratified layer, the buoyancy frequency ${\overline{N^{2}}}^{z}$ averaged over the stratified layer,

which is evaluated by,

$$
{\overline{N^{2}}}^{z}=\frac{1}{H-H_{t b b l}} \int_{H_{t b b l}}^{H} \frac{B_{s} z}{\kappa H} d z=\frac{B_{s}}{2 \kappa}\left(1+\frac{H_{t b b l}}{H}\right),
$$

decreases with the water depth $H$. It is consistent with the model result in Fig.

5b that the scaling (9) predicts larger $H_{t b b l}$ as $H$ increases.

Figure 6 compares the energy conversion rates, $\mathrm{P}^{i}$ - and $\mathrm{B}^{i}$-terms, and the efficiency of tidal mixing $\epsilon$ between the model result and corresponding scaling (Equations (10), (11), and (13)). The $\mathrm{P}^{i}$ - and $\mathrm{B}^{i}$-terms in the model are evaluated by the integration of Equations (22) and (23) over the TBBL, namely,

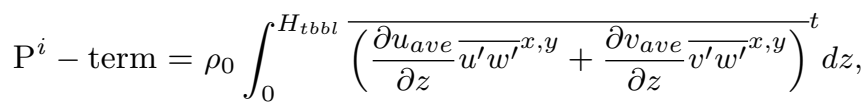

$$
\begin{aligned}
& \mathrm{B}^{i}-\operatorname{term}=-\rho_{0} \int_{0}^{H_{t b b l}}{\overline{b_{V}^{\prime} w^{\prime}}}^{x, y, t} d z .
\end{aligned}
$$

As seen in Figs. $6 \mathrm{a}$ and $6 \mathrm{~b}$, the model $\mathrm{P}^{i}$-term agrees with its scaling $C_{P} u_{*}^{3}$ with $C_{P}=11.6$ and the model $\mathrm{B}^{i}$-term does with its scaling $C_{B} B_{s} H_{t b b l}^{2} / H$ with $C_{B}=$ 0.468 .

The efficiency of tidal mixing $\epsilon$ calculated by $\mathrm{B}^{i}$-term $/ \mathrm{P}^{i}$-term in the model experiment is plotted against the scaling (11) with $C_{\epsilon}^{B P}=C_{B} / C_{P}=0.0403$ in Fig. 6c. Though slightly underestimating the model results for larger Rot (2 and 4) and lower Re (less than 2000) with $H=10 H_{\text {tide }}$ (solid black and red symbols), the scaling (11) shows a fairly good agreement with the model result on the whole.

On the other hand, Figure 6d compares the model $\epsilon$ with the scaling (13) using $C_{\epsilon}\left(3.24 \times 10^{-3}\right)$ evaluated by the best fitted values of $C_{H}(0.7), C_{P}(11.6), C_{B}$ (0.468), and $C_{t b b l}(0.383)$ for the scalings (4), (10), and (7). The model result is 2 more scattered against the scaling (13) than the scaling (11). More specifically, 
whereas the scaling well agrees with the model result when $H=20 H_{\text {tide }}$, it significantly underestimates the model result when $H=10 H_{\text {tide }}$, particularly for $\mathrm{Ro}_{\mathrm{t}}=4$ (solid red symbols). This underestimation appears to be related with the fact that the scaling of $H_{t b b l}(7)$ is smaller than the model result (Fig. 5a). Indeed, the scaling (11) using the model $H_{t b b l}$ gives the better agreement with the model result. Further discussion about discrepancies between the model result and scaling will be given in next subsection.

\subsection{Discussion}

Examining Fig. 6 carefully, we can find that the $\mathrm{P}^{i}$-term is small when the scalings of $\epsilon$ (11) and (13) underestimate the model result (red and black solid symbols). It indicates that the smaller $\mathrm{P}^{i}$-term is a possible cause for the underestimation of $\epsilon$, and then we investigate the reason why the $\mathrm{P}^{i}$-term is smaller in these cases. Figure 7 compares the time evolutions of vertical velocity variance $\left({\overline{w^{2}}}^{x, y}\right)$ and buoyancy anomaly ${\overline{b_{V}}}^{x, y}$ between case 12 with $\left(\operatorname{Rot}, B_{s}\right)=\left(2,1.40 \times 10^{-8} \mathrm{~m}^{2} \mathrm{~s}^{-3}\right)$ and case 21 with $\left(4,2.33 \times 10^{-8} \mathrm{~m}^{2} \mathrm{~s}^{-3}\right)$. Note that $\mathrm{Re}=3000$ and $H=10 H_{\text {tide }}$ in both cases. While the model $\epsilon(1.7 \%)$ is comparable to the scalings $(1.6 \%$ by (11) and $2.0 \%$ by $(13))$ in case 12 , it is larger in case $21(4.9 \%)$ than the scalings $(3.9 \%$ by (11) and $1.4 \%$ by (13)).

In case 12 (Fig. 7a), turbulent motion begins to develop before the background tidal current directs to the major-axis at every half tidal cycle such as $30.0,30.5$, 31.0, and so on, and it continues till the current direction turns to the minor-axis. This long-lived turbulence satisfies the prerequisite to apply the scale argument to the model result on the tidal average. 
In case $21\left(\mathrm{Rot}_{\mathrm{t}}=4\right.$; Fig. $\left.7 \mathrm{~b}\right)$, to the contrary, onset of vigorous turbulence is delayed to the time when the background current turns from the major axis to the minor one such as $21.2,21.7,22.2$, and so on. Turbulence temporarily becomes much more intense than in case 12 , but decays in a short time. Such intermittent and short-lived turbulence which makes the $\mathrm{P}^{i}$-term smaller inherently avoids the scaling based on the tidal average. The $\mathrm{P}^{i}$-term in case $21\left(4.95 \times 10^{-4} \mathrm{Wm}^{-2}\right)$ is less than one third of that in case $12\left(17.6 \times 10^{-4} \mathrm{Wm}^{-2}\right)$ while the $\mathrm{B}^{i}$-term is almost the same $\left(2.44 \times 10^{-5} \mathrm{Wm}^{-2}\right.$ and $2.95 \times 10^{-5} \mathrm{Wm}^{-2}$ in cases 21 and 12 , respectively). It follows that decreased $\mathrm{P}^{i}$-term may be a major factor to increase the efficiency $\epsilon$ in case 21. Similar tendency is found in cases with the short-lived turbulence.

There are some possible reasons why turbulent motion is short-lived, or the $\mathrm{P}^{i}$-term is smaller, in case 21 and others. Higher ellipticity of the background tidal current, i.e. the ratio of the major and minor axes which is equivalent to Rot in the present study, can reduce turbulent intensity when the current directs to the minor axis. The shallower water depth $H$ as well as higher surface heat flux can be another factor. As $H$ decreases, the effective buoyancy flux $B_{s}^{*}$ increases (Equation (5)), and stratification is enhanced in the upper layer (e.g. Equation (26)). These factors effectively suppress turbulent motion, or the $\mathrm{P}^{i}$-term, in the TBBL.

On the other hand, it should be noted that enhanced stratification also act to increase buoyancy anomaly $b_{V}^{\prime}$. Therefore, the $\mathrm{B}^{i}$-term is less reduced or unchanged in cases with the short-lived turbulence although vertical velocity $w^{\prime}$ is suppressed.

Although the model $\mathrm{P}^{i}$-term is small on the tidal average in case 21 , the intermittent turbulent motion itself is much stronger than the long-lived turbulent motion in case 12 (Fig. 7). Indeed, isolines of ${\overline{b_{V}}}^{x, y}$ abruptly rises by more than 1 
m synchronously with the intermittent turbulence (Fig. 7b) whereas its rise is at most a few tenths of a meter in case 12 . This may be a reason why the model $H_{t b b l}$ is larger than that the scaling (7) predicts, particularly for $\mathrm{Rot}_{\mathrm{t}}=4$ and $H=10 H_{\text {tide }}$ (solid red symbols in Fig. 5a). It follows that the scaling of $\epsilon$ (13) underestimates the model result.

Another difference is that the short-lived turbulence begins to develop when the background tidal current turns from the major-axis to the minor whereas the long-lived one begins with the increasing background current velocity. Close examination reveals that the short-lived turbulence is excited by inflection point instability which develops in the decelerating phase as in the Stokes flow which is an oscillating flow without Earth rotation (e.g. Sakamoto and Akitomo, 2006). Lower Re, higher $B_{s}$, and shallower $H$ tend to make turbulence weaker, and finally an inflection point appears in the vertical profile of the horizontal current when the tidal phase proceeds from a flood (ebb) tide to a slack. This is a possible mechanism of short-lived turbulence. Though, to our knowledge, this kind of turbulence has not yet been observed in actual seas, it may be possible when $\operatorname{Ro}_{t}>1$, i.e. Earth rotation is less effective.

Related to the intensity of turbulence in the TBBL, it is meaningful to point out the fact that $u_{*}$ decreases with $B_{s}, H$, and Rot even if $U_{\text {tide }}$ (or Re) is unchanged (Table 1). For example, $u_{*}$ decreases from $5.83 \times 10^{-3} \mathrm{~ms}^{-1}$ to $4.48 \times 10^{-3} \mathrm{~ms}^{-1}$ (a decrease of $25 \%$ ) when $B_{s}$ increases from $0.467 \times 10^{-8} \mathrm{~m}^{2} \mathrm{~s}^{-3}$ to $1.87 \times 10^{-8} \mathrm{~m}^{2} \mathrm{~s}^{-3}$ $10 \mathrm{Wm}^{-2}$ to $40 \mathrm{Wm}^{-2}$ ) in cases $34 \sim 37$. This change is due to the short-lived turbulence occurring more frequently in case 34 than in case 37 (not shown). On the contrary, the decrease in $u_{*}$ is only $5 \%$ in cases $11 \sim 14$ where no short-lived turbulence occurs. Nevertheless, the scaling law presented here is still valid when 


\section{Summary}

We have investigated what determines the height of the TBBL $H_{t b b l}$ in a shallow sea under a constant heating by scale argument and DNS experiment. Different from other boundary layers, it is peculiar to the TBBL that inputs of the buoyancy and turbulent kinetic energy are located on the opposite ends of the water layer. Because of this, the buoyancy flux at the top of the TBBL, i.e. the effective buoyancy flux, is reduced by a factor of $H_{t b b l} / H$ from that at the sea surface, where $H$ is the water depth (Fig. 1).

Taking it into account, we have obtained the scaling of $H_{t b b l}$ under a constant heating $B_{s}$ for the first time. That is,

$$
H_{t b b l}=u_{*} / \sqrt{C_{H}^{-2}(\sigma+f)^{2}+C_{N S}^{-2}|\sigma+f| B_{s} H_{t b b l} / H u_{*}^{2}} .
$$

This scaling is reduced to that for a homogeneous sea $H_{h o m}=C_{H} u_{*} /|\sigma+f|$ (Sakamoto and Akitomo, 2008) when $B_{s}$ is set to zero, or the water depth is sufficiently large relative to $H_{\text {hom }}$. In a shallow coastal sea where the water depth is usually much smaller than $H_{h o m}, H_{t b b l}$ can be approximated to

$$
H_{t b b l} \approx C_{t b b l}\left(\frac{u_{*}^{4} H}{|\sigma+f| B_{s}}\right)^{1 / 3}
$$

${ }^{02}$ where $C_{t b b l}\left(\equiv C_{N S}^{2 / 3}\right)$ is the empirical constant. $H_{t b b l}$ increases with $H$ because increasing $H$ reduces the effective buoyancy flux by putting the top of the TBBL away from the heating source at the sea surface. Using the scaling of $H_{t b b l}$, we 
can obtain a simple expression for the efficiency of the energy conversion from the tidal turbulent kinetic energy to the potential energy of the water column, $\epsilon$, as

$$
\epsilon=C_{\epsilon} \frac{H_{h o m}}{H_{t b b l}} .
$$

DNS experiment exhibits a good agreement with these scalings of the TBBL with $C_{t b b l}=0.383$, and the efficiency with $C_{\epsilon}=3.24 \times 10^{-3}$ although there are some exceptional cases with slow Earth's rotations $\left(\mathrm{Ro}_{t}=2\right.$ and 4$)$, weak tidal flows $(\operatorname{Re} \leq 2000)$, and shallow water depth $\left(H=10 H_{\text {tide }}\right)$. In the exceptional cases, the scaling argument fails because intermittent and short-lived turbulent motion avoids assumption of tidal average. Therefore, the proposed scalings can be good measures of turbulent properties of the TBBL under a surface heating as long as the tidal average is physically meaningful. We expect that advanced observation technologies will reveal fine-scale turbulent properties to validate the present results.

When the TBBL reaches the sea surface, the efficiency $\epsilon$ is given by $C_{\epsilon} H_{h o m} / H$. This means that the critical value of SH parameter $\log \left(H / U^{3}\right)$ which is an index of the position of tidal mixing front may increase with $u_{*}\left(U_{\text {tide }}\right)$ and decrease with $|\sigma+f|$ and $H$. The variation range of $\epsilon$ from less than 1 to $7 \%$ in the present experiment implies that the critical value of $\mathrm{SH}$ parameter may differ by nearly one, which is comparable to the observed difference around the world (e.g. Simpson and Sharples, 2012). A comprehensive review of observational data should be needed about variability of the efficiency of tidal mixing.

A diurnal variation of surface heating and the horizontal component of Earth's rotation (Wakata, 2013) may modify the present results by introducing temporal 
${ }_{445}\left(u_{\text {int }}(t), v_{\text {int }}(t)\right)$ with negative $f$ is obtained as,

$$
\left(u_{\text {int }}(t), v_{\text {int }}(t)\right)=\left(-U_{\text {tide }} \cos \sigma t,-V_{\text {tide }} \sin \sigma t\right)
$$
where $U_{\text {tide }}$ and $\sigma$ are the amplitude and frequency of the forcing, respectively, ${ }_{447}$ and $V_{\text {tide }}=U_{\text {tide }}(f / \sigma)$ is the amplitude of $v_{\text {int }}(t)$. Using complex number $(i$ : 448 the imaginary unit), this expression is converted to a sum of clockwise and anti- 
449 clockwise components:

$$
u_{i n t}(t)+i v_{i n t}(t)=R^{+} e^{i \sigma t}+R^{-} e^{-i \sigma t}
$$

$$
u_{t i d e}(z, t)+i v_{t i d e}(z, t)
$$

$$
=\left\{\begin{array}{rr}
R^{+} e^{i \sigma t}\left(1-\exp \left(-\frac{(1+i) z}{\sqrt{2 \nu /(f+\sigma)}}\right)\right)+R^{-} e^{-i \sigma t}\left(1-\exp \left(-\frac{(1+i) z}{\sqrt{2 \nu /(f-\sigma)}}\right)\right) \\
\text { when } \quad f+\sigma>0 \quad \text { and } \quad f-\sigma>0 \\
R^{+} e^{i \sigma t}\left(1-\exp \left(-\frac{(1+i) z}{\sqrt{2 \nu /(f+\sigma)}}\right)\right)+R^{-} e^{-i \sigma t}\left(1-\exp \left(-\frac{(1-i) z}{\sqrt{2 \nu /(f-\sigma)}}\right)\right) \\
\text { when } \quad f+\sigma>0 \quad \text { and } \quad f-\sigma<0 \\
R^{+} e^{i \sigma t}\left(1-\exp \left(-\frac{(1-i) z}{\sqrt{2 \nu /(f+\sigma)}}\right)\right)+R^{-} e^{-i \sigma t}\left(1-\exp \left(-\frac{(1+i) z}{\sqrt{2 \nu /(f-\sigma)}}\right)\right) \\
\text { when } \quad f+\sigma<0 \quad \text { and } \quad f-\sigma>0 \\
R^{+} e^{i \sigma t}\left(1-\exp \left(-\frac{(1-i) z}{\sqrt{2 \nu /(f+\sigma)}}\right)\right)+R^{-} e^{-i \sigma t}\left(1-\exp \left(-\frac{(1-i) z}{\sqrt{2 \nu /(f-\sigma)}}\right)\right) \\
\text { when } \quad f+\sigma<0 \quad \text { and } \quad f-\sigma<0 .
\end{array}\right.
$$

where $R^{ \pm}=-\left(U_{\text {tide }} \pm V_{\text {tide }}\right) / 2$ (double-sign corresponds). Now, imposing equation (30) as the boundary condition at $z \rightarrow \infty$ while no-slip condition at the bottom, we obtain the analytical solution of $u_{\text {tide }}(z, t)+i v_{t i d e}(z, t)$ for the viscous fluid with constant $\nu$,

Acknowledgements The authors acknowledge Dr. Y. Yoshikawa for his discussion. This study was partly supported by the TEAMS (Tohoku Ecosystem-Associated Marine Sciences) from MEXT (Ministry of Education, Culture, Sports, Science, and Technology of Japan). Numerical calculation was carried out on the supercomputer of ACCMS, Kyoto University. 


\section{References}

1. Awaji T, Imasato N, Kunishi H (1980) Tidal exchange through a strait: a numerical experiment using a simple model basin. J. Phys. Oceanogr., 10, 1499-1508.

2. Coleman G N (1999) Similarity statistics from a direct numerical simulation of the neutrally stratified planetary boundary layer. J. Atmos. Sci. 56:891-900.

3. Davies A M (1985) On determining current profiles in oscillatory flows. Appl. Math. Model. $9: 419-428$

4. Fang G, Ichiye T (1983) On the vertical structure of tidal currents in a homogeneous sea. Geophys. J. R. Astr. Soc. 73:65-82.

5. Garrett C J R, Keeley J R, Greenberg D A (1978) Tidal mixing versus thermal stratification in the Bay of Fundy and the Gulf of Maine. Atmospheres and Ocean 16: 403-423.

6. Glorioso P D, Flather R A (1995) A barotropic model of the currents off SE South America. Journal of Geophysical Research 100: 13427-13440.

7. Kobayashi S, Simpson J H, Fujiwara T, Horsburgh K J (2006) Tidal stirring and its impact on water column stability and property distributions in a semi-enclosed shelf sea (Seto Inland Sea, Japan) Continental Shelf Research 26: 1295-1306

8. Lie H J (1989) Tidal fronts in the south-eastern Hwanghae (Yellow Sea). Continental Shelf Research 9: 527-546

9. Pingree R D, Griffiths D K (1978) Tidal fronts on the shelf seas around the British Isles. Journal of Geophysical Research 83: 4615-4622.

10. Sakamoto K, Akitomo K (2006) Instability of the tidally induced bottom boundary layer in the rotating frame and their mixing effect. Dyn Atmos Oceans 41:191-211

11. Sakamoto K, Akitomo K (2008) The tidally induced bottom boundary layer in a rotating frame: similarity of turbulence. J Fluid Mech 615:1-25

12. Sakamoto K, Akitomo K (2009) The tidally induced bottom boundary layer in the rotating frame: development of the turbulent mixed layer under stratification. J Fluid Mech 619:235259

13. Sakamoto K, Tsujino H, Nakano H, Hirabara M, Yamanaka G (2013a) A practical scheme to introduce explicit tidal forcing into OGCM. Ocean Sci Discussions 10(2):473-517 
14. Sakamoto K, Yamanaka G, Tsujino H, Nakano H, Hirabara M (2013b) Development of a $2 \mathrm{~km}$-resolution Japanese coastal ocean model -toward a next-generation operational monitoring and forecasting system (in Japanese), Weather Service Bulletin, 80, S99-S109.

15. Simpson J H, Hunter J R (1974) Fronts in the Irish Sea. Nature 250:404-406

16. Simpson J H, Sharples J (2012) Introduction to the physical and biological oceanography of shelf seas. 448 pp. Cambridge University Press, Cambridge.

17. Sun Y-J, Isobe A (2008) Lagrangian $\mathrm{H} / \mathrm{U}^{3}$ values computed around fronts using a fineresolution numerical model and ferryboat-monitored SST dataset. J. Phys. Oceanogr., 38: $2575-2586$.

18. Takeoka H, Kaneda A, Anami H (1997) Tidal fronts induced by horizontal contrast of vertical mixing efficiency. Journal of Oceanography 53: 563-570.

19. Tsutsumi E, Matsuno T (2012) Observations of turbulence under weakly and highly stratified conditions in the Ariake Sea. Journal of Oceanography 68: 369-386.

20. Wakata Y (2013) Some properties of tidal currents estimated from analytical and LES simulation studies. Journal of Oceanography 69: 737-751

21. Yanagi T, Tamaru H (1990) Temporal and spatial variations in a tidal front. Continental Shelf Research 10: 615-627.

22. Yoshikawa Y (2015) Scaling Surface Mixing/Mixed Layer Depth under Stabilizing Buoyancy Flux. J. Phys. Oceanogr., 45, 247-258.

23. Zilitinkevich S, Esaub I, Baklanovc A (2007) Further comments on the equilibrium height of neutral and stable planetary boundary layers. Q. J. R. Meteorol. Soc. 133: 265-271. 


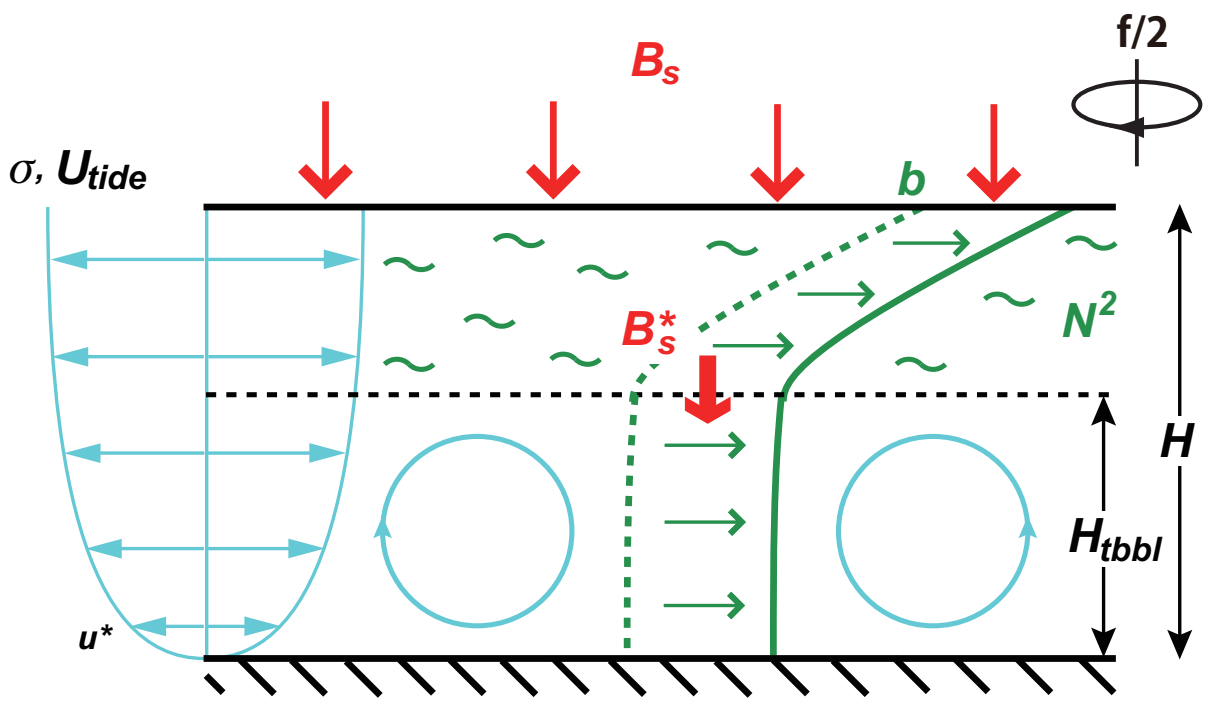

Fig. 1 Schematic view of tidally-induced bottom boundary layer under a surface heating. See text for detail. 


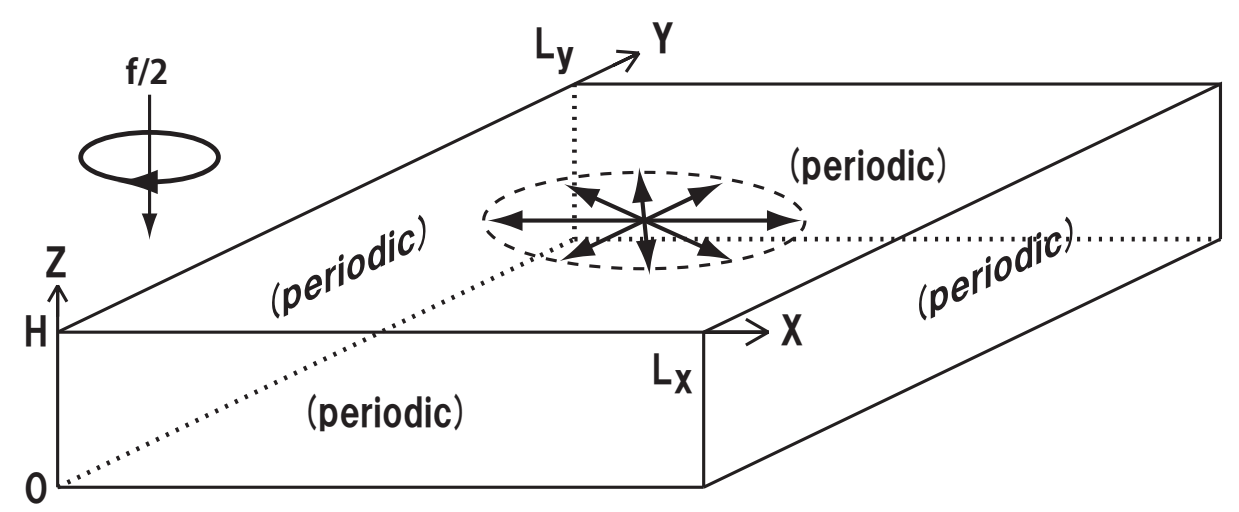

Fig. 2 Model domain and coordinate system with tidal flow as the driving force. 


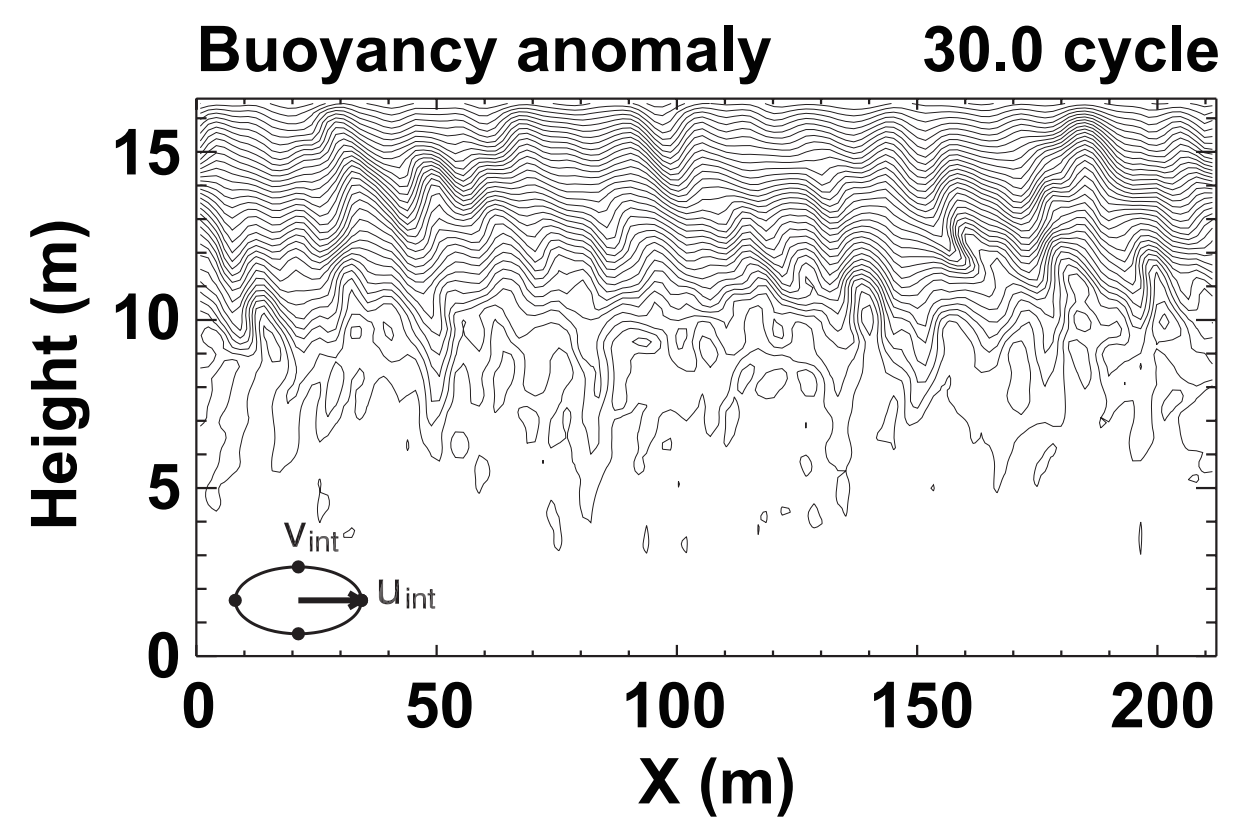

Fig. 3 Vertical section of buoyancy anomaly $b_{V}^{\prime}$ at $y=106 \mathrm{~m}$ on 30 tidal cycles. Contour interval is $2.0 \times 10^{-5} \mathrm{~m} \mathrm{~s}^{-2}$. The ellipse of interior tidal flow is shown as the forcing with the vector pointing the flow direction. 
(a)

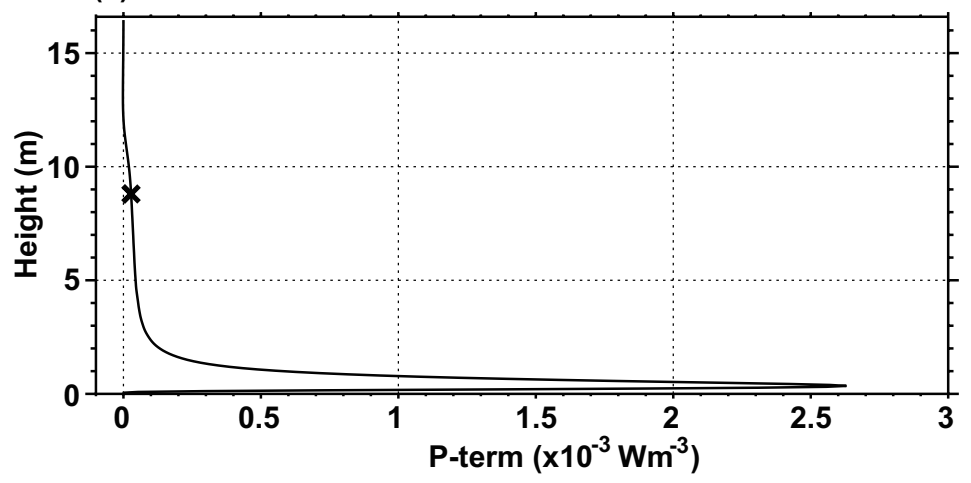

(b)

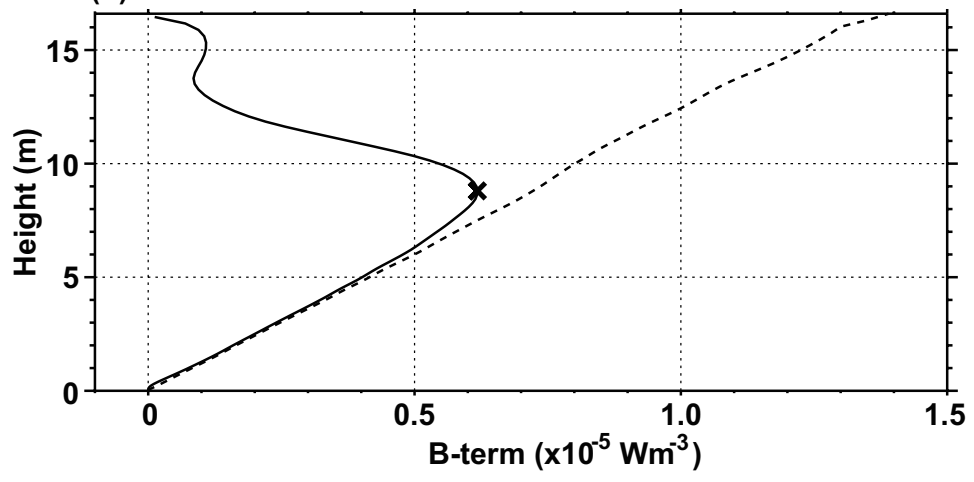

(c)

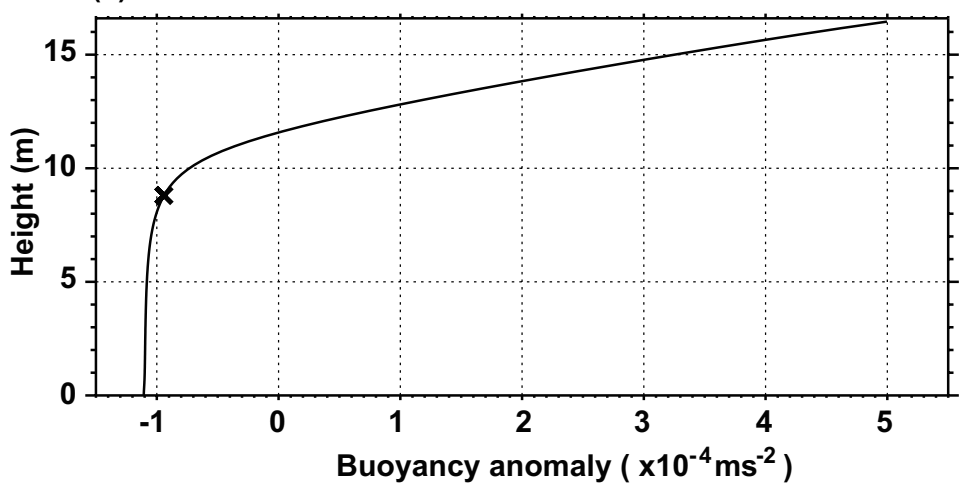

Fig. 4 Solid lines indicate vertical profiles of (a) $\mathrm{P}-$ term, (b) $\mathrm{B}-$ term, and (c) ${\overline{b_{V}}}^{x, y, t}$. Cross $(x)$ indicates the height where $\mathrm{B}-$ term reaches the maximum, or the height of TBBL, $H_{t b b l}$. Dashed line in (b) shows the horizontal and temporal average of the total buoyancy flux, $B_{f}$. 
(a)

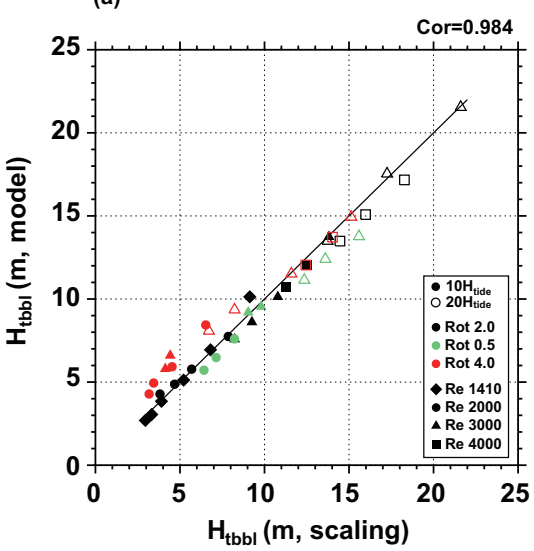

(b)

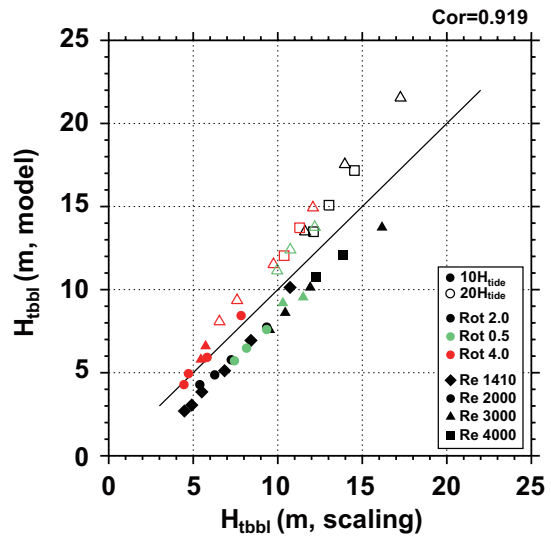

Fig. 5 (a) $H_{t b b l}$ evaluated from model result against the scaling given by Equation (7) with $C_{t b b l}=0.383$, (b) same as in (a) but for the scaling given by Equation (9) with $C_{C N}=1.56$. Solid and open symbols represent cases with $H=10 H_{\text {tide }}$ and $20 H_{\text {tide }}$, respectively; black, green, and red ones do cases with $\mathrm{Ro}_{\mathrm{t}}=2,0.5$, and 4, respectively; diamond, circle, triangle, and square do cases with $\mathrm{Re}=1410,2000,3000$, and 4000, respectively. 
(a)

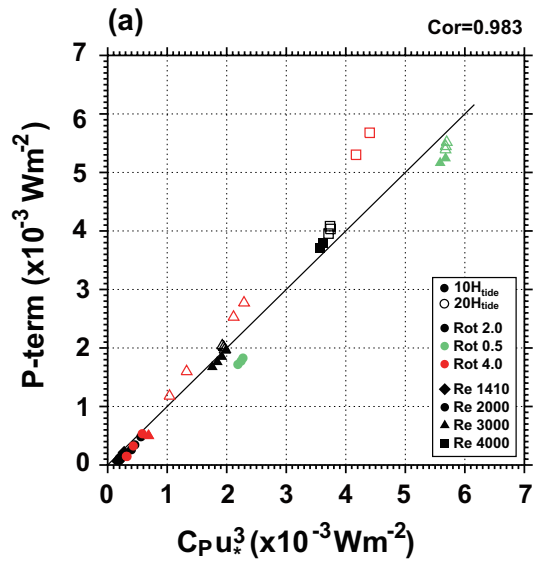

(c)

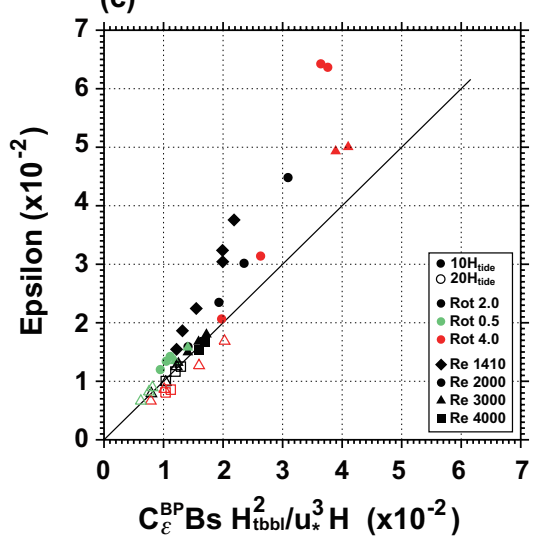

(b)

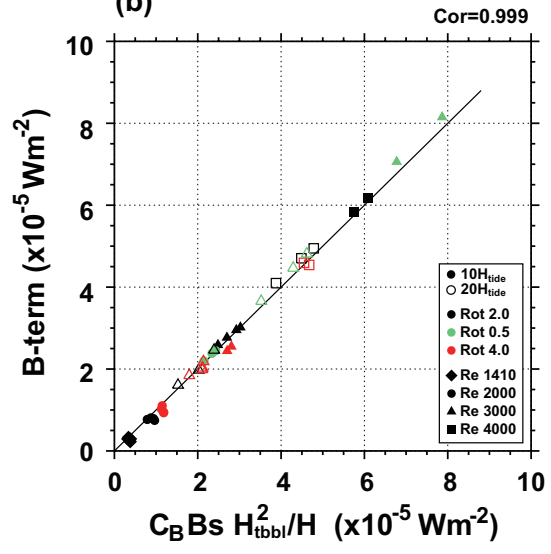

(d)

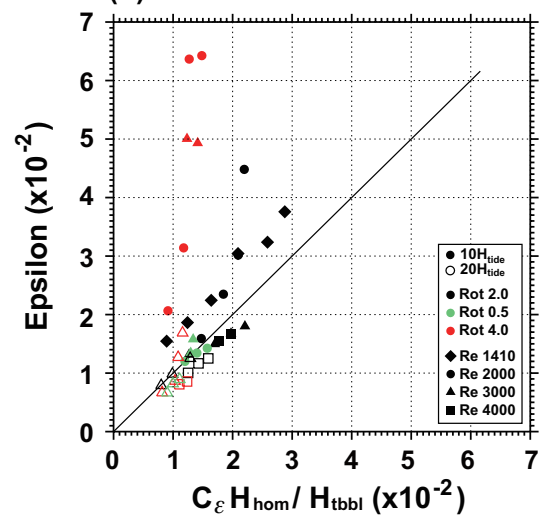

Fig. 6 (a) $\mathrm{P}^{i}$-term evaluated from model experiment against the scaling given by Equation (10) with $C_{P}=11.6$, (b) $\mathrm{B}^{i}$-term evaluated from model experiment against the scaling given by Equation (10) with $C_{B}=0.468$, (c) $\epsilon$ evaluated from model experiment against the scaling given by Equation (11) with $C_{\epsilon}^{B P}=C_{B} / C_{P}=0.0403$, and (d) same as in (c) but for the scaling given by Equation (13) with $C_{\epsilon}=C_{t b b l}^{3} C_{B} / C_{H} C_{P}=3.24 \times 10^{-3}$. Symbols are same as in Fig. 5. 

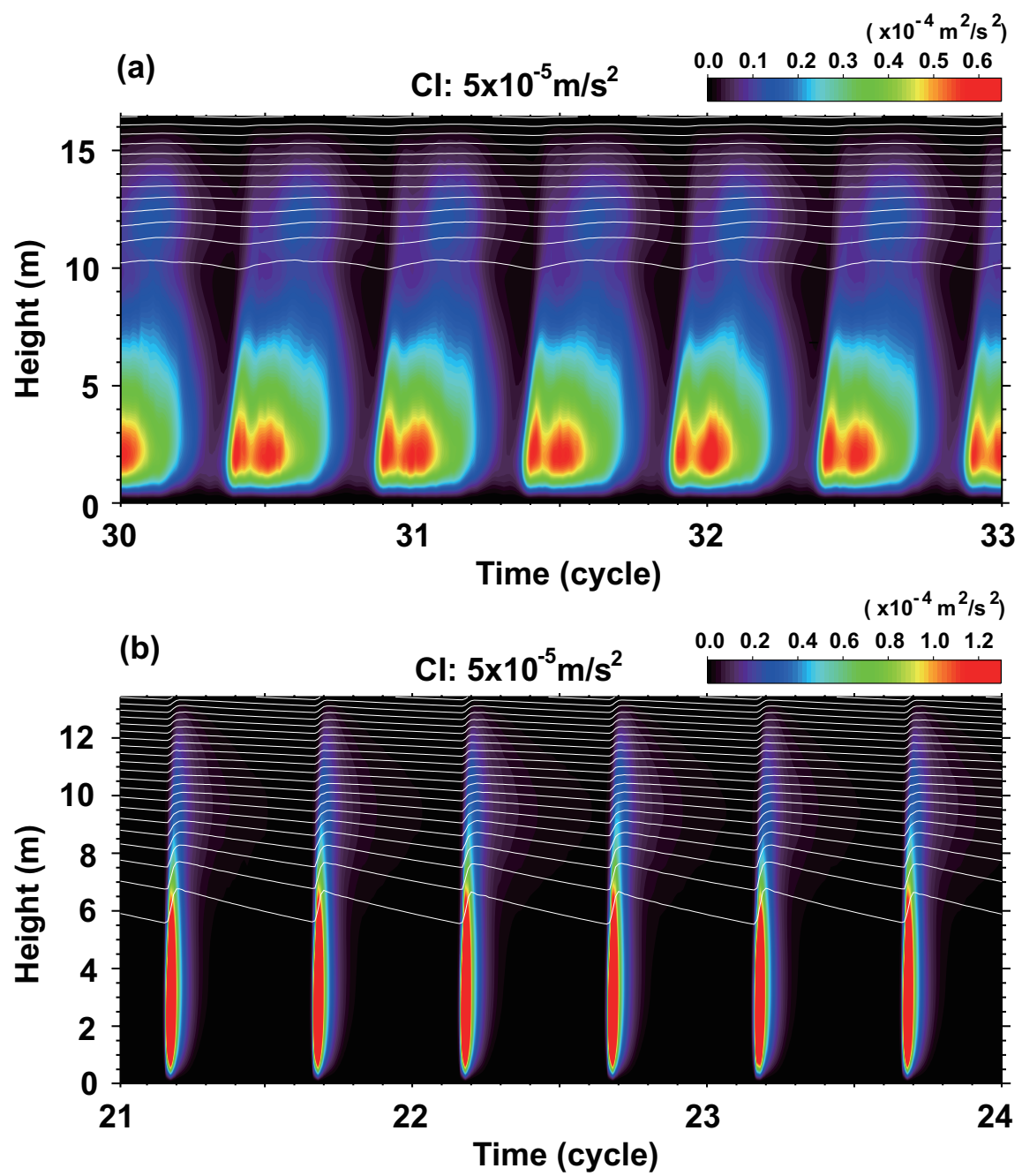

Fig. 7 Time evolution of variance ${\overline{w^{2}}}^{x, y}$ (colors) and buoyancy anomaly ${\overline{b_{V}}}^{x, y}$ (white contours). (a) Case 12, and (b) case 21. Contour intervals are shown in each panel. Note that color contour interval in (b) is doubled that in (a). 
Table 1 Experimental cases

\begin{tabular}{|c|c|c|c|c|c|c|c|c|}
\hline Case & $\begin{array}{c}U_{\text {tide }} \\
\left(\mathrm{ms}^{-1}\right)\end{array}$ & $\begin{array}{r}H_{\text {tide }} \\
(\mathrm{m})\end{array}$ & $\begin{array}{c}H \\
\left(\times H_{\text {tide }}\right)\end{array}$ & $\begin{array}{c}B_{s} \\
\left(\times 10^{-8} \mathrm{~m}^{2} \mathrm{~s}^{-3}\right)\end{array}$ & $\begin{array}{c}f \\
\left(\times 10^{-4} \mathrm{~s}^{-1}\right)\end{array}$ & $\begin{array}{c}\operatorname{Re} \\
\left(U_{\text {tide }} H_{\text {tide }} / \nu\right)\end{array}$ & $\begin{array}{c}\mathrm{Rot}_{\mathrm{t}} \\
(|\sigma / f|)\end{array}$ & $\begin{array}{c}u_{*} \\
\left(\times 10^{-2} \mathrm{~ms}^{-1}\right)\end{array}$ \\
\hline 1 & 0.0850 & 1.66 & 10 & 1.87 & -0.727 & 1410 & 2 & 0.248 \\
\hline 2 & 0.0850 & 1.66 & 10 & 1.40 & -0.727 & 1410 & 2 & 0.252 \\
\hline 3 & 0.0850 & 1.66 & 10 & 0.933 & -0.727 & 1410 & 2 & 0.257 \\
\hline 4 & 0.0850 & 1.66 & 10 & 0.467 & -0.727 & 1410 & 2 & 0.268 \\
\hline 5 & 0.0850 & 1.66 & 10 & 0.233 & -0.727 & 1410 & 2 & 0.275 \\
\hline 6 & 0.0850 & 1.66 & 10 & 0.117 & -0.727 & 1410 & 2 & 0.289 \\
\hline 7 & 0.121 & 1.66 & 10 & 1.87 & -0.727 & 2000 & 2 & 0.300 \\
\hline 8 & 0.121 & 1.66 & 10 & 1.40 & -0.727 & 2000 & 2 & 0.326 \\
\hline 9 & 0.121 & 1.66 & 10 & 0.933 & -0.727 & 2000 & 2 & 0.340 \\
\hline 10 & 0.121 & 1.66 & 10 & 0.467 & -0.727 & 2000 & 2 & 0.365 \\
\hline 11 & 0.181 & 1.66 & 10 & 1.87 & -0.727 & 3000 & 2 & 0.534 \\
\hline 12 & 0.181 & 1.66 & 10 & 1.40 & -0.727 & 3000 & 2 & 0.543 \\
\hline 13 & 0.181 & 1.66 & 10 & 0.933 & -0.727 & 3000 & 2 & 0.550 \\
\hline 14 & 0.181 & 1.66 & 10 & 0.467 & -0.727 & 3000 & 2 & 0.557 \\
\hline 15 & 0.241 & 1.66 & 10 & 1.87 & -0.727 & 4000 & 2 & 0.677 \\
\hline 16 & 0.241 & 1.66 & 10 & 1.40 & -0.727 & 4000 & 2 & 0.679 \\
\hline 17 & 0.148 & 1.35 & 10 & 1.87 & -0.364 & 2000 & 4 & 0.304 \\
\hline 18 & 0.148 & 1.35 & 10 & 1.40 & -0.364 & 2000 & 4 & 0.301 \\
\hline 19 & 0.148 & 1.35 & 10 & 0.933 & -0.364 & 2000 & 4 & 0.334 \\
\hline 20 & 0.148 & 1.35 & 10 & 0.467 & -0.364 & 2000 & 4 & 0.369 \\
\hline 21 & 0.222 & 1.35 & 10 & 2.33 & -0.364 & 3000 & 4 & 0.392 \\
\hline 22 & 0.222 & 1.35 & 10 & 1.87 & -0.364 & 3000 & 4 & 0.390 \\
\hline 23 & 0.171 & 1.17 & 10 & 1.87 & -2.91 & 2000 & 0.5 & 0.575 \\
\hline 24 & 0.171 & 1.17 & 10 & 1.40 & -2.91 & 2000 & 0.5 & 0.579 \\
\hline 25 & 0.171 & 1.17 & 10 & 0.933 & -2.91 & 2000 & 0.5 & 0.582 \\
\hline 26 & 0.256 & 1.17 & 10 & 2.33 & -2.91 & 3000 & 0.5 & 0.785 \\
\hline 27 & 0.256 & 1.17 & 10 & 1.87 & -2.91 & 3000 & 0.5 & 0.790 \\
\hline 28 & 0.181 & 1.66 & 20 & 0.933 & -0.727 & 3000 & 2 & 0.554 \\
\hline 29 & 0.181 & 1.66 & 20 & 0.467 & -0.727 & 3000 & 2 & 0.553 \\
\hline 30 & 0.181 & 1.66 & 20 & 0.233 & -0.727 & 3000 & 2 & 0.551 \\
\hline 31 & 0.241 & 1.66 & 20 & 1.87 & -0.727 & 4000 & 2 & 0.685 \\
\hline 32 & 0.241 & 1.66 & 20 & 1.40 & -0.727 & 4000 & 2 & 0.687 \\
\hline 33 & 0.241 & 1.66 & 20 & 0.933 & -0.727 & 4000 & 2 & 0.687 \\
\hline 34 & 0.222 & 1.35 & 20 & 1.87 & -0.364 & 3000 & 4 & 0.448 \\
\hline 35 & 0.222 & 1.35 & 20 & 1.40 & -0.364 & 3000 & 4 & 0.486 \\
\hline 36 & 0.222 & 1.35 & 20 & 0.933 & -0.364 & 3000 & 4 & 0.568 \\
\hline 37 & 0.222 & 1.35 & 20 & 0.467 & -0.364 & 3000 & 4 & 0.583 \\
\hline 38 & 0.295 & 1.35 & 20 & 1.87 & -0.364 & 4000 & 4 & 0.713 \\
\hline 39 & 0.295 & 1.35 & 20 & 1.40 & -0.364 & 4000 & 4 & 0.725 \\
\hline 40 & 0.256 & 1.17 & 20 & 1.87 & -2.91 & 3000 & 0.5 & 0.789 \\
\hline 41 & 0.256 & 1.17 & 20 & 1.40 & -2.91 & 3000 & 0.5 & 0.790 \\
\hline 42 & 0.256 & 1.17 & 20 & 0.933 & -2.91 & 3000 & 0.5 & 0.790 \\
\hline
\end{tabular}

